2013

\title{
Neutral Depletion And The Helicon Density Limit
}

R. M. Magee

M.E. Galante

J. Carr

G. Lusk

D. W. McCarren

See next page for additional authors

Follow this and additional works at: https://researchrepository.wvu.edu/faculty_publications

\section{Digital Commons Citation}

Magee, R. M.; Galante, M. E.; Carr, J.; Lusk, G.; McCarren, D. W.; and Scime, E. E., "Neutral Depletion And The Helicon Density Limit" (2013). Faculty Scholarship. 610.

https://researchrepository.wvu.edu/faculty_publications/610 
Authors

R. M. Magee, M. E. Galante, J. Carr, G. Lusk, D. W. McCarren, and E. E. Scime 


\title{
Neutral depletion and the helicon density limit
}

\author{
R. M. Magee, ${ }^{\text {a) }}$ M. E. Galante, J. Carr, Jr., G. Lusk, D. W. McCarren, and E. E. Scime \\ West Virginia University, Morgantown, West Virginia 26506, USA
}

(Received 31 May 2013; accepted 2 December 2013; published online 19 December 2013)

\begin{abstract}
It is straightforward to create fully ionized plasmas with modest rf power in a helicon. It is difficult, however, to create plasmas with density $>10^{20} \mathrm{~m}^{-3}$, because neutral depletion leads to a lack of fuel. In order to address this density limit, we present fast $(1 \mathrm{MHz})$, time-resolved measurements of the neutral density at and downstream from the rf antenna in krypton helicon plasmas. At the start of the discharge, the neutral density underneath the antenna is reduced to $1 \%$ of its initial value in $15 \mu$ s. The ionization rate inferred from these data implies that the electron temperature near the antenna is much higher than the electron temperature measured downstream. Neutral density measurements made downstream from the antenna show much slower depletion, requiring $14 \mathrm{~ms}$ to decrease by a factor of $1 / e$. Furthermore, the downstream depletion appears to be due to neutral pumping rather than ionization. (C) 2013 AIP Publishing LLC. [http://dx.doi.org/10.1063/1.4849376]
\end{abstract}

\section{INTRODUCTION}

Helicons ${ }^{1-3}$ are typically composed of two distinct regions: a plasma source and an expansion chamber. The plasma source is where the rf power is coupled to the fuel gas and the plasma is created. The expansion chamber is a separate volume usually located several $10 \mathrm{~s}$ of $\mathrm{cm}$ downstream from the source. It is in this volume that the plasma is put to work; it is typically where measurements are made, samples are inserted, or thrust is generated. It is therefore in this region, downstream from the antenna, that maximizing plasma density will most benefit future industrial application.

We recently showed that the ionization fraction in the expansion chamber of our krypton helicon plasmas can, under the right conditions, reach $100 \% .{ }^{4}$ The plasma density, however, is usually only $1 \%-10 \%$ of the initial fill density. Figure 1 shows that in krypton plasmas with an initial fill pressure of $4 \mathrm{mT}$, the efficiency (defined here to be the ratio of the electron density to initial neutral density, $\left.n_{e} / n_{0, i}\right)$ plateaus at $8 \%$ around $1 \mathrm{~kW}$ and does not improve with even a five-fold increase in rf power. This low efficiency represents an ultimate limit to the plasma density.

It is clear then that the observed high ionization levels are due not to complete ionization, but to neutral depletion, because if ionization was the only process removing neutrals from the measurement volume, the measured plasma density would equal the initial fill density. It is not clear, however, what is causing the depletion. The object of the present work is to quantify the dynamics of neutral depletion in the helicon to address this question and suggest pathways to improve the overall efficiency.

We present fast $(1 \mathrm{MHz})$, time-resolved two photon absorption laser induced fluorescence (TALIF) measurements of the neutral density in both the source and expansion region of krypton helicon plasmas. The measurements are interpreted in conjunction with Langmuir probe measurements of the plasma density to construct a complete picture

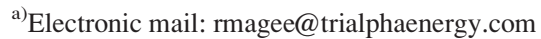

of the dynamics of the plasma and neutral gas in the helicon discharge and to offer a conjecture for the density limit illustrated in Figure 1. We begin with a brief discussion of previous work upon which the present work is built.

\section{PREVIOUS WORK}

The problem of fueling from neutral gas injected at the plasma boundary is well known in magnetic fusion energy research. In the tokamak, where high electron densities and temperatures at the edge prevent neutrals from penetrating beyond a few centimeters, core fueling via the injection of cryogenic pellets or high energy neutral beams is required. ${ }^{5}$

In low temperature plasmas, the mean free path of ionization is usually longer than the size of the plasma, so the dynamics of the neutrals and the plasma are more tightly coupled and can affect one another in non-intuitive ways. For example, it has been shown both theoretically and experimentally that neutral depletion in low temperature rf plasmas can lead to an increase in plasma transport, resulting in a decreasing plasma density with increasing input power. ${ }^{6,7}$ This result can be understood as follows: as the input power is increased, ionization increases plasma density at the expense of neutral density. But as the neutral density decreases, the neutral drag on ions also decreases, and ions are able to escape the plasma volume more quickly. The net result is a lower plasma density despite a higher ionization rate. A similar phenomenon was reported in an experiment which employed two antennas to increase the deposited power. ${ }^{8}$ Again, the regions of plasma with the highest ionization rates were also the regions with the highest transport, which limited the overall achievable density.

The examples above describe cases in which changes to the neutral density profile affect the plasma density profile, but the reverse also occurs. Neutral pumping generally refers to a process in which neutrals are removed from a plasma through some mechanism other than ionization. There are at least two such mechanisms commonly cited. The first is the expulsion of neutrals due to non-ionizing collisions with ions. This occurs when the plasma pressure becomes 


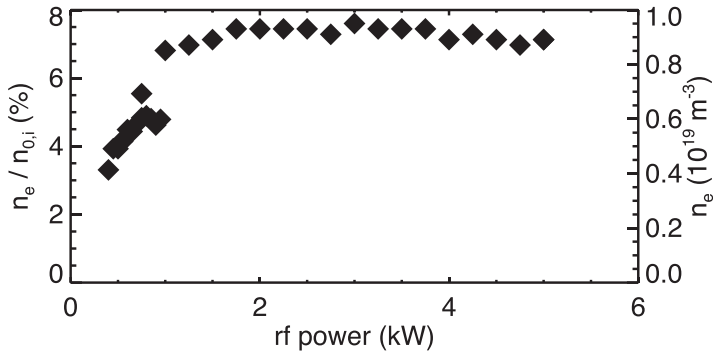

FIG. 1. The ionization efficiency in the expansion chamber of a krypton plasma with an initial fill pressure of $4 \mathrm{mT}$ as a function of rf power plateaus around $1 \mathrm{~kW}$. The right $\mathrm{y}$-axis shows the corresponding absolute plasma density.

comparable to the neutral gas pressure, but the plasma temperature remains relatively low. In order for radial force balance to be maintained, neutrals must be convected out of regions of high plasma pressure., ${ }^{2,9,10}$ The second is the process in which neutrals are ionized and accelerated out of the plasma by an electric field faster than they are replenished by fueling. ${ }^{11}$ This electric field can take the form of a sheath electric field or an internal electric field (produced in a double layer, for example). The accelerated ions can then charge exchange with edge neutrals and leave the plasma. Because the velocity of these escaping charge exchange neutrals is much larger than the velocity of the inflowing neutrals (which are moving at the neutral thermal velocity), the influx is smaller than the out flux, and the overall neutral inventory is depleted.

\section{COMPACT HELICON AND DIAGNOSTICS}

\section{A. Compact helicon}

The experiments were conducted in the compact helicon shown in Figure 2. The plasma is generated in a Pyrex tube

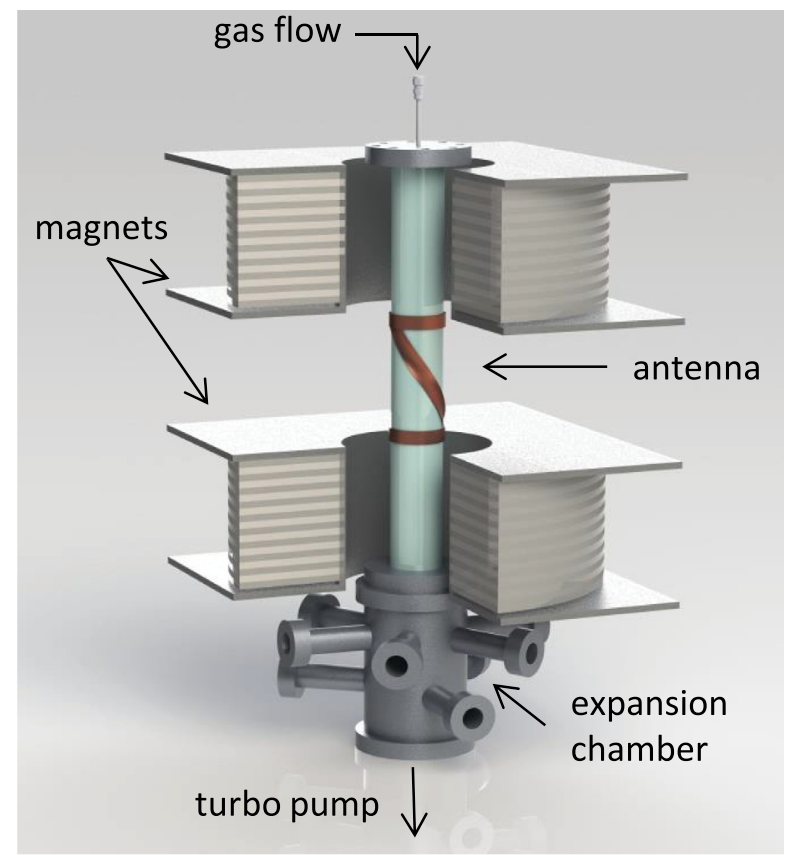

FIG. 2. The compact helicon. Measurements are made in the expansion chamber and underneath the antenna. Reprinted with permission from Phys. Plasmas 19, 123506 (2012). Copyright (C) 2012 AIP Publishing LLC. ${ }^{4}$ with $D=5 \mathrm{~cm}$ and $L=60 \mathrm{~cm}$ by applying $0.1-5 \mathrm{~kW}$ of $\mathrm{rf}$ power with $f=13.56 \mathrm{MHz}$ to an $m=1$ Shoji type helical antenna. The gas is fed from the top via a mass flow controller and pumped from below with a turbomolecular drag pump. The fueling and pumping rates remain constant throughout the experiment. All data presented here were obtained with krypton as the fuel. The Pyrex tube is coupled to a stainless steel expansion chamber with $D=14 \mathrm{~cm}$ and $L=25 \mathrm{~cm}$ and 9 portholes for diagnostic access.

To make time-resolved measurements, the rf power is square wave modulated. A Caesar $1350 \mathrm{RF}$ Generator is pulsed with an external signal generator at a frequency of $20 \mathrm{~Hz}$ (to match the repetition rate of the laser). The pulse length is varied from 10 to $25 \mathrm{~ms}$ (i.e., duty cycle $20 \%-50 \%$ ), and the rise time of the rf power is less than $0.1 \mu \mathrm{s}$. The delay between the leading edge of the trigger pulse for the rf and the laser is manually adjusted so that the time history of the signal is built up over many discharges. In subsequent discussion, $t=0$ refers to the rf turn on time. The effect of the pulse length on the neutral density is discussed in Sec. IV C.

\section{B. TALIF}

The principle diagnostic is the TALIF system. It is composed of a high power (1 MW peak), UV $(205 \mathrm{~nm})$ pulsed laser operated at $20 \mathrm{~Hz}$. The laser is capable of producing $8 \mathrm{~ns}$ pulses with energy $E=8 \mathrm{~mJ}$, although for the present experiment the energy was reduced to $0.5 \mathrm{~mJ}$ to avoid saturation. The laser light excites an electronic transition in neutral krypton, from the $4 p$ (ground) to $5 p$ state. De-excitation emission is collected and coupled to a photomultiplier tube, high speed amplifier, and boxcar averager. The details of the diagnostic have been presented elsewhere. ${ }^{12}$ Measurements from within the expansion chamber (Figure 6) were obtained by injecting the laser into one of the 9 portholes, and the stimulated emission is coaxially collected from the same port. Measurements from the source (Figures 3, 5, 8-10) were taken with the source and expansion chamber rotated by $90^{\circ}$ and the laser injected and emission collected from what is shown as the top port in Figure 2.

\section{Time-resolved Langmuir probe measurements}

We use a rf-compensated, ${ }^{13}$ cylindrical tip $(0.5 \mathrm{~mm}$ diameter graphite) Langmuir probe to make the measurements of plasma density. A bias voltage is applied between

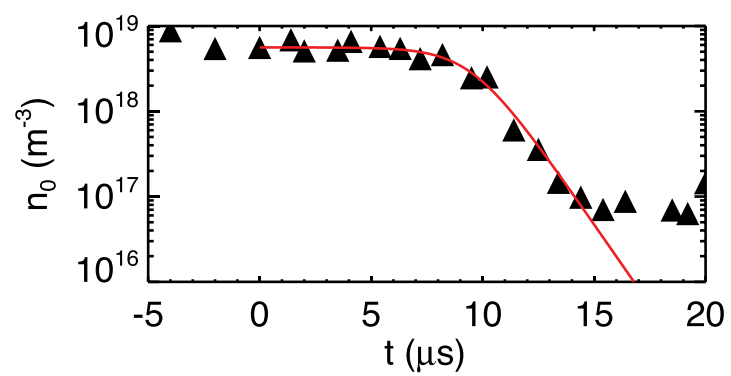

FIG. 3. The neutral density in a $4.5 \mathrm{~kW}$ plasma measured in the source. The rf power is turned on at $t=0$. The solution to the coupled differential equations described in the text is overlaid in red. 


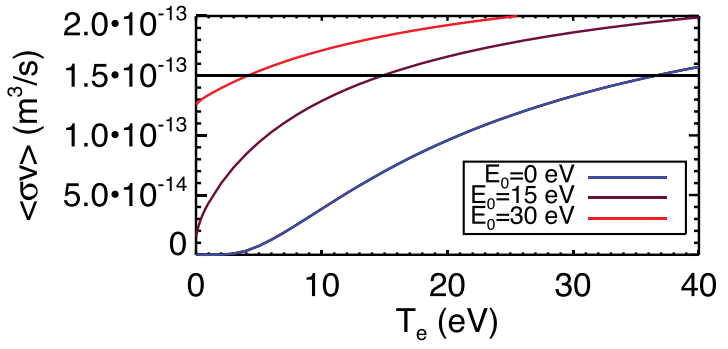

FIG. 4. The curves are the calculated ionization rates as functions of temperature using idealized Maxwell-Boltzmann distributions with 3 different flow velocities and the values of $\sigma(E)$ tabulated by Lotz. The horizontal line is the ionization rate given by the fit to the data.

the probe tip and electrical ground with a DC power supply. The resulting current is determined from measurements of the voltage drop across a $150 \Omega$ resistor made with a custom built differential amplifier and an oscilloscope with a data acquisition rate of $50 \mathrm{kS} / \mathrm{s}$. As with the TALIF measurement, time resolution is achieved by pulsing the rf power supply of the plasma. In this case, a given bias voltage is applied to the probe, and the time histories of the voltage traces from several discharges are averaged together. The bias is manually scanned from $-40 \mathrm{~V}$ to $+25 \mathrm{~V}$ to build the $\mathrm{I}-\mathrm{V}$ curve.

\section{RESULTS}

\section{A. Ionization}

The first results presented are measurements of the neutral density as a function of time in the plasma source (see Figure 3). These data are taken underneath the antenna in a $\mathrm{rf}=4.5 \mathrm{~kW}$ plasma with an axial magnetic field $B=1200 \mathrm{G}$, an initial fill pressure of $4 \mathrm{mT}$, and a rf duty cycle of $20 \%$. The neutral density decays on a very short timescale (1-10 $\mu \mathrm{s})$. The thermal velocity of a room temperature krypton neutral is $170 \mathrm{~m} / \mathrm{s}$, so it takes $2 \mathrm{~ms}$ for a neutral to travel the $30 \mathrm{~cm}$ from the gas inlet to the antenna. It is therefore reasonable to neglect the inflowing neutrals when constructing a model of the time behavior of the neutral density. It is also reasonable to hypothesize that the timescales are too short for the neutral pumping mechanisms mentioned above to be active. To test this hypothesis, we consider ionization only and compare the implications to the experimental data.

If electron impact ionization is the only process depleting neutrals and creating electrons, the neutral and electron

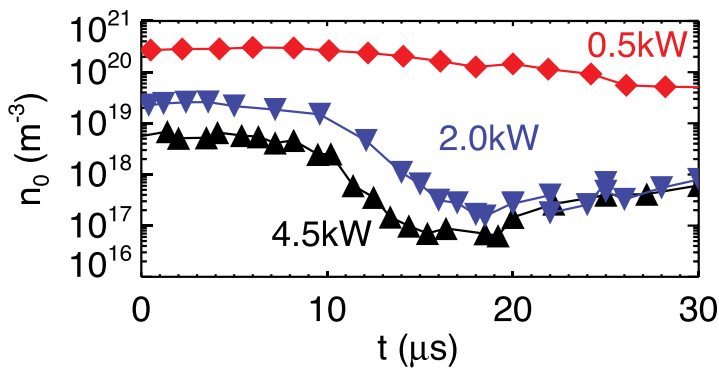

FIG. 5. The neutral density plotted as a function of time for 3 different $\mathrm{rf}$ powers. In all cases, the initial fill density was the same $\mathrm{P}=5 \mathrm{mT}$, corresponding to $n_{0}=1.5 \times 10^{20} \mathrm{~m}^{-3}$. The $4.5 \mathrm{~kW}$ case is same as that presented in Figure 3.

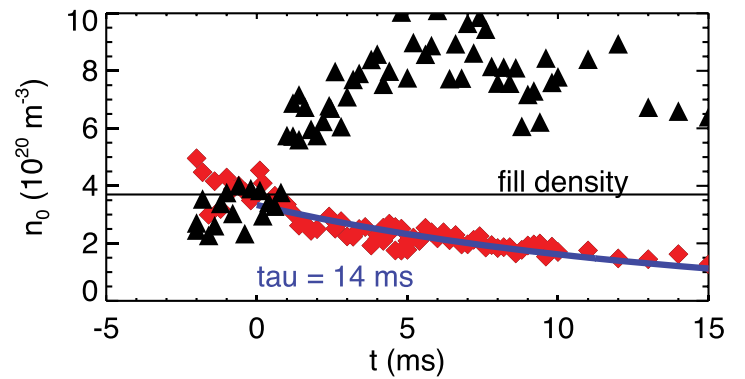

FIG. 6. The neutral density in the expansion chamber $(z=75 \mathrm{~cm})$ as a function of time. The red diamonds are the data taken from $r=0 \mathrm{~cm}$ and the black triangles from $r=6.7 \mathrm{~cm}$. The blue curve is the result of fitting the data to a simple exponential.

densities as functions of time are governed by the coupled differential equations

$$
\begin{aligned}
& \frac{d n_{0}}{d t}=-\langle\sigma v\rangle_{E I} n_{0} n_{e}, \\
& \frac{d n_{e}}{d t}=+\langle\sigma v\rangle_{E I} n_{0} n_{e},
\end{aligned}
$$

where $\langle\sigma v\rangle_{E I}$ is the electron impact ionization rate. We assume that the total number of particles is constant in time, $n_{0}(t)+n_{e}(t)=n_{t o t}$, and we allow there to exist a small initial population of primary electrons, $n_{e, i}$. The neutral density as a function of time is then given by

$$
n_{0}(t)=\frac{n_{0, i}}{1-A \exp \left(n_{t o t}\langle\sigma v\rangle_{E I} t\right)},
$$

where $n_{0, i}$ is the initial density of neutrals and $A \equiv\left(1-n_{\text {tot }} / n_{0, i}\right)$.

We fit Eq. (3) to the measured data, allowing $n_{e, i}$ (via $A$ ) and $\langle\sigma v\rangle_{E I}$ to be fit parameters. The result of the Levenberg-Marquardt least-squares fit is overlaid in red. The fit is quite good over two decades of neutral density, an indication that the assumption that ionization is the dominant process early in time is valid. The values for the free parameters are $n_{e, i}=2.5 \times 10^{-4} n_{0, i}$ and $\langle\sigma v\rangle_{E I}=1.5 \times 10^{-13} \mathrm{~m}^{3} \mathrm{~s}^{-1}$.

We can compare the ionization rate given by the fit to the data and the value of $\langle\sigma v\rangle_{E I}$ calculated from tabulated values of $\sigma(E)$ using idealized electron energy distributions. Using the empirical formula given by Lotz for $\sigma(E),{ }^{14}$ we find that the inferred ionization rate is consistent with an electron temperature, $T_{e}=37 \mathrm{eV}$.

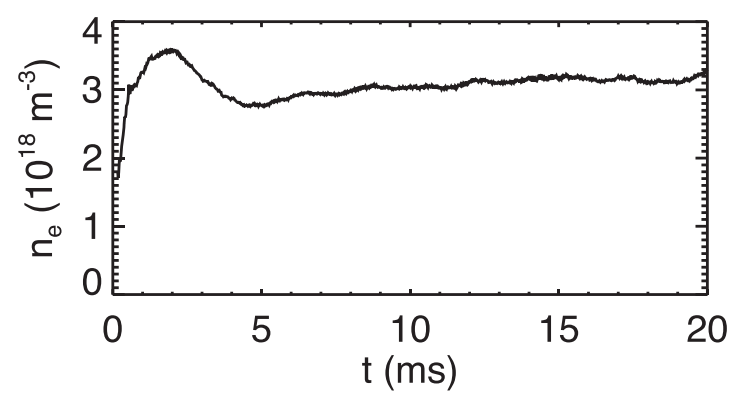

FIG. 7. The electron density as a function of time as measured in the expansion chamber with a Langmuir probe. The electron density reaches a steady state in about $5 \mathrm{~ms}$, much more quickly than the neutral density. 


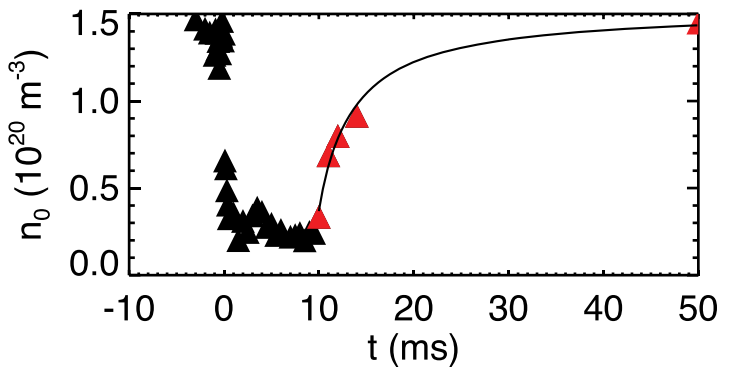

FIG. 8. The neutral density in the source in a $0.5 \mathrm{~kW}$ plasma shows the relatively slow rate of neutral replenishment after the rf is turned off at $t_{\text {off }}=10 \mathrm{~ms}$. Data points taken after this time are shown in red and the black line is a fit to these points only.

Electron temperature data from underneath the antenna are not available, but $T_{e}=37 \mathrm{eV}$ is considerably higher than values typically measured in the expansion chamber, where $T_{e} \sim 5-10 \mathrm{eV}$. However, if we allow for a bulk flow of electrons, due to an axial electric field, for example, lower temperatures are required (in other words, we will use a shifted Maxwellian for the electron energy distribution, rather than one centered at $v=0$ ). For example, a distribution centered at $E_{0}=15 \mathrm{eV}$ (corresponding to a bulk electron flow, $v_{0}=5.8 \mathrm{~km} / \mathrm{s}$ ) only needs $T_{e}=15 \mathrm{eV}$ to match the inferred ionization rate. For $E_{0}=30 \mathrm{eV}\left(v_{0}=8.3 \mathrm{~km} / \mathrm{s}\right), T_{e}=5 \mathrm{eV}$. Figure 4 shows the inferred ionization rate (horizontal line) and the ionization rates as functions of electron temperature for the 3 above mentioned flow velocities.

Allowing for a bulk electron flow is not enough to resolve the discrepancy between the high inferred ionization rate and the electron temperature measured downstream, because the required flow for the $T_{e}=5 \mathrm{eV}$ case is larger than reasonable expectation. Mach probe data taken in the expansion chamber during an earlier experiment show maximum flows of $v=2 \mathrm{~km} / \mathrm{s}$. ${ }^{12}$ There are then really only two (non-mutually exclusive) interpretations of these data: the average electron energy is higher in the plasma source than in the expansion region or the distribution function is not Maxwellian such that there is a disproportionate number of high energy electrons. We note that it has been postulated before that a small population of suprathermal electrons created by interaction with the rf are responsible for the bulk of the ionizing collisions. ${ }^{3}$ The present analysis however is unable to distinguish between these hypotheses, and, in fact, it may be the case that there are distinct electron energy

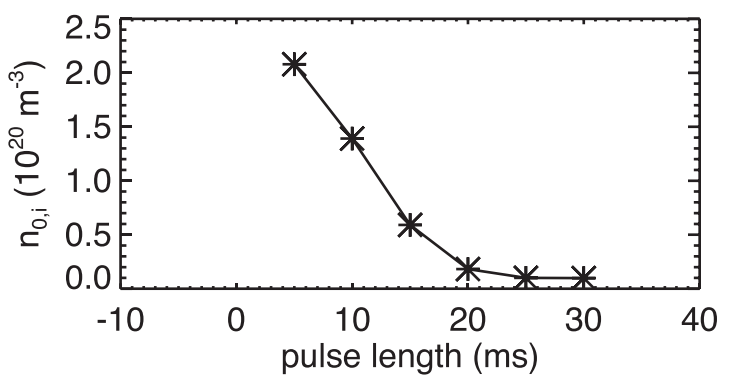

FIG. 9. The neutral density at $t=0$ is plotted as a function of pulse length for a $20 \mathrm{~Hz}$ repetition rate. As the pulse length is increased, the time between pulses becomes shorter than the recombination time, and the initial neutral density drops.

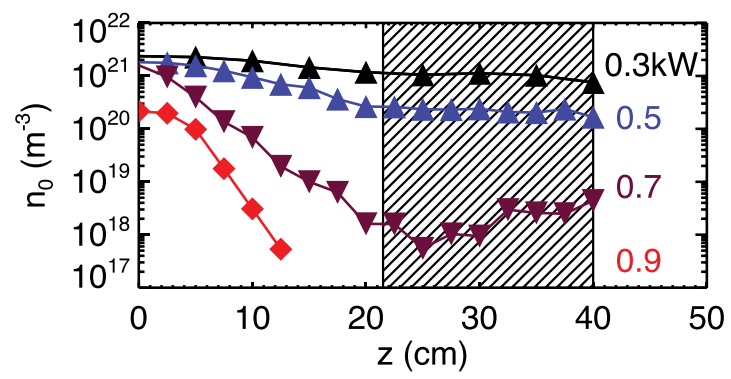

FIG. 10. The CW equilibrium neutral density as a function of axial position for 4 different rf powers. The shaded region represents the antenna.

distributions upstream and downstream and that the upstream distribution is non-Maxwellian.

Shown in Figure 5 are time histories of the absolute neutral density for three different rf powers. In all three cases, the fueling rate was $0.6 \mathrm{sccm}$ to give an initial fill pressure of $5 \mathrm{mT}$ and the magnetic field strength was $1200 \mathrm{G}$. It can be seen that increasing the power from $0.5 \mathrm{~kW}$ to $2.0 \mathrm{~kW}$ increases the ionization rate, presumably due to an increase in the average electron energy. Increasing the power further to $4.5 \mathrm{~kW}$ does not increase the rate. It does, however, result in a decrease of the initial $(t=0)$ neutral density value. The persistent feature that in pulsed plasmas the neutral density does not return to the initial fill density at the start of the discharge is due to the fact that the replenishment time is longer than the pulse period. This phenomenon is discussed quantitatively in Sec. IV C.

\section{B. Neutral pumping}

Measurements of the neutral density in the expansion chamber as a function of time show a strikingly different picture from those taken in the source. Measurements made in the core and at the edge of the expansion chamber are plotted in Figure 6. This region is approximately $45 \mathrm{~cm}$ downstream from the center of the antenna $(z=75 \mathrm{~cm}$ in Figure 10). The plasma in this case had $\mathrm{rf}=600 \mathrm{~W}$ and an initial fill pressure $P=4 \mathrm{mT}$. The depletion is much slower than the depletion observed in the source, and there are two indications that it is not due to ionization.

The first indication comes from the observation that as the neutral density is depleted in the core, it rises at the edge. This is consistent with neutral particles being convected radially outward. The second indication comes from Langmuir probe measurements made at the same location in similar plasmas. They show that the core electron density reaches a steady state much more quickly than the neutral density. As seen in Figure 7 , the electron density reaches $3 \times 10^{18} \mathrm{~m}^{-3}$ in about $1 \mathrm{~ms}$. If the slow neutral depletion measured in the core was due to ionization, we would expect the electron density to increase on the same timescale. Here, again we see that the equilibrium plasma density is much smaller than the original fill density. In fact, it turns out to be smaller by a factor roughly equal to the ratio of the volume of the source region to the volume of the expansion chamber, 1/40th.

We now offer a conjecture for the chronology of events in the formation of our helicon plasma. When the rf is turned on, a small volume of gas in the antenna region is completely 
ionized in $15 \mu \mathrm{s}$, and the electron density reaches a value equal to the initial fill density in the same time. This localized, small volume of high density plasma diffuses into the expansion region. As the plasma expands, the density and the temperature drop, and the additional neutral gas encountered is not efficiently ionized. The gas does however react to the plasma pressure, as the two are now comparable $\left(P_{e}=1.4-4.8 \mathrm{~Pa}\right.$ for $n_{e}=3 \times 10^{18} \mathrm{~m}^{-3}$ and $T_{e}=3-10 \mathrm{eV}$ and $P_{n}=1.6-6.4 \mathrm{~Pa}$ for $n_{0}=4 \times 10^{20} \mathrm{~m}^{-3}$ and $\left.T_{0}=0.025-0.1 \mathrm{eV}\right)$. The neutrals are expelled from the core and accumulate at the edge due to ion-neutral collisions.

This picture also offers an explanation for the efficiency limit described in Sec. I. Although the plasma in the expansion chamber is fully ionized, it is not because all of the available gas there has been ionized. The available gas has been expelled by the plasma pressure, and the density of the plasma is determined by expansion of the fully ionized source into the expansion chamber.

We emphasize that the two paragraphs above are purely conjecture. Complementary measurements of the electron density in the source are required to test this hypothesis.

\section{Neutral replenishment}

At the end of the discharge the rf power is turned off (the magnetic field remains on) and the neutrals are replenished. This presumably occurs due to a combination of influxing neutrals and plasma recombination. The measured neutral replenishment is shown in Figure 8. The data from $t>t_{\text {off }}$ (shown in red) are well fit by the function $n_{0}(t)=$ $n_{\text {tot }}-10^{20} /(\alpha t+C)$ with $\alpha=0.24$ and $C=0.86$. The fit is overlaid in black. (Note that the rf power is $0.5 \mathrm{~kW}$ in Figure 8 , whereas it is $4 \mathrm{~kW}$ in Figure 3 . This is why the minimum neutral density is $0.2 n_{0, i}$ here and $0.01 n_{0, i}$ in Figure 3.)

This slow replenishment time has a very practical consequence. If the time between rf pulses is shorter than the replenishment time, the $t=0$ neutral density drops over the course of several pulses, until it reaches a new value which is less than the initial fill density. This effect can be seen in Figure 9 where we plot the neutral density at $t=0$ as a function of pulse length. The repetition rate is held constant at $20 \mathrm{~Hz}$, so as the pulse length is increased, the time between pulses decreases. Therefore, to maximize the plasma density, the time between pulses should be increased until the initial neutral density is equal to the initial fill density.

\section{Equilibria}

If the rf power is kept below $1 \mathrm{~kW}$, the source can be operated in $\mathrm{CW}$ mode. Measurements of the axial profiles in these plasmas show dramatic neutral depletion and very large neutral density gradients (see Figure 10). For context, we note that the density in the $0.7 \mathrm{~kW}$ case drops by 3 orders of magnitude in $20 \mathrm{~cm}$, a gradient comparable to that of neutral hydrogen in the edge of the DIII-D tokamak. ${ }^{15}$

\section{CONCLUSION}

Time-resolved measurements of the neutral density at multiple spatial locations reveal new details of the dynamics of the formation of a helicon discharge. At the start of the discharge, the neutral density underneath the rf antenna is almost completely depleted in $15 \mu$ s. The inferred ionization rate is faster than expected given the electron energy distribution measured downstream. This could imply that the bulk of the ionization is accomplished by a small population of very energetic electrons (perhaps those energized by the large rf fields), or that the upstream and downstream electron energy distributions are different and kept from achieving thermal equilibrium. Measurements of the radial profile of the neutral density in the expansion chamber, downstream from the antenna, show a decrease in the core density on a much slower, $10 \mathrm{~ms}$, timescale. The coincident accumulation of neutrals at the edge and Langmuir probe measurements, which show much faster electron dynamics, suggest that this depletion is due not to ionization but to neutral expulsion. The possibility that the fully ionized plasma in the expansion chamber arises from the downstream convected plasma expelling neutrals may influence the design of future helicons capable of producing high densities throughout.

\section{ACKNOWLEDGMENTS}

This work is funded by the U.S. Department of Energy through Grant No. DE-SC0004736.

${ }^{1}$ R. W. Boswell, "Plasma production using a standing helicon wave," Phys. Lett. A 33, 457-458 (1970).

${ }^{2}$ R. W. Boswell, "Very efficient plasma generation by whistler waves near the lower hybrid frequency," Plasma Phys. Controlled Fusion 26, 1147-1162 (1984).

${ }^{3}$ F. F. Chen, "Plasma ionization by helicon waves," Plasma Phys. Controlled Fusion 33, 339-364 (1991).

${ }^{4}$ R. M. Magee, M. E. Galante, N. Gulbrandsen, D. W. McCarren, and E. E. Scime, "Direct measurements of the ionization profile in krypton helicon plasmas," Phys. Plasmas 19, 123506 (2012).

${ }^{5}$ J. Wesson, Tokamaks (Claredon Press, Oxford, 2004).

${ }^{6}$ A. Fruchtman, G. Makrinich, P. Chabert, and J. M. Rax, "Enhanced plasma transport due to neutral depletion," Phys. Rev. Lett. 95, 115002 (2005).

${ }^{7}$ L. Liard, J.-L. Raimbault, J.-M. Rax, and P. Chabert, "Plasma transport under neutral gas depletion conditions," J. Phys. D: Appl. Phys. 40, 5192-5195 (2007).

${ }^{8}$ D. G. Miljak and F. F. Chen, "Density limit in the helicon," Plasma Sources Sci. Technol. 7, 537-549 (1998).

${ }^{9}$ A. Aanesland, L. Liard, G. Leray, J. Jolly, and P. Chabert, "Direct measurements of neutral density depletion by two-photon absorption laser-induced fluorescence spectroscopy," Appl. Phys. Lett. 91, 121502 (2007).

${ }^{10}$ L. Liard, A. Aanesland, and P. Chabert, "Dynamics of neutral gas depletion investigated by time- and space-resolved measurements of xenon atom ground state density," J. Phys. D: Appl. Phys. 45, 235201 (2012).

${ }^{11}$ J. Gilland, R. Breun, and N. Hershkowitz, "Neutral pumping in a helicon discharge,” Plasma Sources Sci. Technol. 7, 416-422 (1998).

${ }^{12}$ R. M. Magee, M. E. Galante, D. McCarren, E. E. Scime, R. L. Boivin, N. H. Brooks, R. J. Groebner, D. N. Hill, and G. D. Porter, "A two photon absorption laser-induced fluorescence diagnostic for fusion plasmas," Rev. Sci. Instrum. 83, 10D701 (2012).

${ }^{13}$ I. D. Sudit and F. F. Chen, "RF compensated probes for high-density discharges," Plasma Source. Sci. Technol. 3, 162 (1994).

${ }^{14} \mathrm{~W}$. Lotz, "Electron-impact ionization cross-sections and ionization rate coefficients for atoms and ions," Astrophys. J. Suppl. 14, 207-238 (1967).

${ }^{15}$ R. J. Colchin, R. Maingi, M. E. Fenstermacher, T. N. Carlstrom, R. C. Isler, L. W. Owen, and R. J. Groebner, "Measurements of neutral density near the X-point in the DIII-D tokamak," Nucl. Fusion 40, 175-180 (2000). 